\begin{tabular}{cc|c}
\hline Tarım Bilimleri Dergisi & Journal of Agricultural Sciences \\
& $\begin{array}{c}\text { Dergi web sayfası: } \\
\text { www.agri.ankara.edu.tr/dergi }\end{array}$ & Journal homepage: \\
& www.agri.ankara.edu.tr/journal
\end{tabular}

\title{
Agricultural Performances of Some Safflower (Carthamus tinctorius L.) Lines Developed by Single Plant Selection Method
}

\author{
Arzu KÖSE ${ }^{\text {a }}$, Ferda KOŞAR ${ }^{a}$, Özlem BİLİR ${ }^{b}$ \\ ${ }^{a}$ Transitional Zone Agricultural Research Institute, Eskisehir, TURKEY

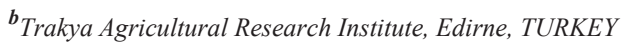

\section{ARTICLE INFO}

Research Article

DOI: 10.15832 /ankutbd.446334

Corresponding Author: Arzu KÖSE, E-mail: arzukose.tr@gmail.com, Tel: +90 (532) 5432608

Received: 24 June 2015, Received in Revised Form: 15 April 2016, Accepted: 14 July 2016

\begin{abstract}
This study was carried out to determine seed and oil yield with their components of some safflower lines and varieties under Eskisehir ecological conditions. This research was conducted at the experimental fields of Transitional Zone Agricultural Research Institute (TZARI), during 2010, 2011 and 2012. Both twenty-one safflower lines and four varieties (Yenice, Dincer, Remzibey and Balci) originating from TZARI-Eskisehir were evaluated in this research. The lines used in this study are developed by using single plant selection method. Three years data were collected and analyzed according to randomized block design with three replications. Means of seed yield, number of head per plant, head diameter, 1000 seed weight, oil content, oil yield were found 1330.3-1990.9 and $1210.1 \mathrm{~kg} \mathrm{ha}^{-1}, 11.2-12.3$ and 9.6 number plant ${ }^{-1}, 2.28-2.42$ and $2.54 \mathrm{~cm}, 41.6-45.7$ and $44.1 \mathrm{~g}, 36.1-36.6$ and 35.6\%, 470.9-730.0 and $430.0 \mathrm{~kg} \mathrm{ha}^{-1}$ in 2010, 2011 and 2012, respectively. According to all years and combined analysis results of this study, lines GE-ESYA-36-36, GE-ES-YA-36-7 in terms of seed yield, lines GE-ES-YA-36-30, GE-ES-YA-36-25, GE-ES-YA-36-26, GEES-YA-36-27 in terms of oil content and lines GE-ES-YA-36-36, GE-ES-YA-36-7, GE-ES-YA-36-4 terms of oil yield were listed at the highest statistical group. As a result of this study, it was decided that these lines could be candidate varieties with regard to these different characteristics.
\end{abstract}

Keywords: Safflower; Selection; Lines; Seed yield; Oil yield

\section{Tek Bitki Seleksiyonu Islahı ile Geliştirilmiş Bazı Aspir (Carthamus tinctorius L.) Hatlarının Tarımsal Performansları}

\section{ESER BILGISII}

Araştırma Makalesi

Sorumlu Yazar: Arzu KÖSE, E-posta: arzukose.tr@gmail.com, Tel: +90 (532) 5432608

Geliş Tarihi: 24 Haziran 2015, Düzeltmelerin Gelişi: 15 Nisan 2016, Kabul: 14 Temmuz 2016

\section{ÖZET}

Bu çalışma, Eskişehir ekolojik koşulları altında, bazı aspir hat ve çeşitlere ait tane ve yağ verimleri ile komponenetlerinin belirlenmesi amacı ile yürütülmüştür. Araştırma, 2010, 2011 ve 2012 yıllarında, Geçit Kuşağı Tarımsal Araştırma 
Enstitüsü (GKTAE) deneme tarlalarında gerçekleştirilmiştir. Çalışmada, GKTAE-Eskişehir tarafından geliştirilmiş 21 adet hat ve 4 standart çeşit (Yenice, Dincer, Remzibey ve Balcı) kullanılmıştır. Çalışmada kullanılan hatlar tek bitki seleksiyon ıslahı yöntemi ile elde edilmiştir. 3 yıla ait veriler, tesadüf blokları deneme desenine uygun olarak analiz edilmiştir. Araştırmada, ortalama tane verimi, bitkide tabla sayısı, tabla çapı, 1000 tane ağırlı̆̆ verimi değerleri 2010, 2011 ve 2012 yıllarında sirasıyla, $1330.3-1990.9$ ve $1210.1 \mathrm{~kg} \mathrm{ha}^{-1}, 11.2-12.3$ ve 9.6 adet bitki-1, 2.28-2.42 ve $2.54 \mathrm{~cm}, 41.6-45.7$ ve $44.1 \mathrm{~g}, 36.1-36.6$ ve $35.6 \%$, 470.9-730.0 ve $430.0 \mathrm{~kg} \mathrm{ha}^{-1}$ olarak bulunmuştur. Yıllara ve birleştirilmiş analiz sonuçlarına göre; tane verimi bakımından GE-ES-YA-36-36, GE-ES-YA-36-7, yağ oranı bakımından GE-ES-YA-36-30, GE- ES-YA-36-25, GE- ES-YA-36-26, GE-ES-YA-36-27, yağ verimi bakımından ise; GE-ES-YA-36-36, GE-ES-YA-36-7, GE-ES-YA-36-4 hatları istatistiki olarak ilk grupta yer almıştır. Çalışma sonuçlara göre, bu hatların belirlenen farklı özellikler bakımından aday çeşit olabileceği sonucuna varılmıştır.

Anahtar Kelimeler: Aspir; Seleksiyon; Hat; Tane verimi; Yağ verimi

(C) Ankara Üniversitesi Ziraat Fakültesi

\section{Introduction}

Turkey is undergoing an increasing loss of foreign exchange for many years due to the ongoing oil deficit in every year. To solve this problem, it is necessary to add other oilseed crop in production, beside of the increase yield of existing oilseed crops. Safflower has capable of wide adaptation, high drought resistance and can grow in arid areas (Omidi et al 2012; El-Lattief 2012). Safflower oil is of great importance as well as important for human nutrition as a raw material for biodiesel (Bergman \& Charles 2008; Mündel 2008; Sujatha 2008; Uher 2008). Taken into consideration cultivation requests of safflower, it can be cultivating easily. It is a suitable plant applied to fallow and wheat cultivating systems in Turkey (Kose et al 2011).

In our country, although safflower production area was about 2,000 ha in 1970 's, it gradually decreases about 30-40 ha at the beginning of the 2000's (TSI 2014). This situation has negative effect of the safflower breeding programs in Turkey. In last year, recognizing the importance of the safflower cause to increase production and breeding researches. According to data, in Turkey, safflower harvested area was 29,259 ha, production quantity is 45,000 tons and yield $1,530 \mathrm{~kg} \mathrm{ha}^{-1}$, which was above the world yield average (FAO 2014).

Breeding program, in accordance with the different objectives, is designed for developing new varieties and is used different breeding method. Selection is one of the oldest breeding procedures and is the basis of all crop improvement. Essentially, selection is a process, either natural or artificial, by which individual plants or groups of plants are sorted out from mixed population (Poehlman 1978). Nowadays, single plant selection method is mostly used in safflower breeding program (Fernandez Martinez et al 1986). In this method single plants are selected according to the morphological and quality parameters from population until obtaining breeding objectives.

This study aimed to estimate the performances of 21 safflower lines, which are used in this study are developed by using single plant selection method as compared to varieties for releasing new safflower varieties.

\section{Material and Methods}

Field trials were performed in 2010, 2011 and 2012 by using 21 safflower lines and 4 varieties (Yenice, Dincer, Remzibey and Balci) developed by Transitional Zone Agricultural Research Institute ( $\left.39^{\circ} 45^{\prime} " 57^{\prime} \mathrm{N}, 30^{\circ} 24^{\prime \prime} 5^{\prime} \mathrm{E}\right)$ in Turkey.

These lines derived from 4 different population P-010, P-029, P-101, P-201 originated from Turkey, Balikesir; China, Qinghai; Turkey, Eskisehir; Turkey Isparta, respectively. The efficiency of a selection program mainly depends on the degree of genetic variation and heritability of a trait (Falconer \& Mackay 1981; Shinwari et al 2014). A significant level of phenotypic variation was noticed among the population for most of the characters. Lines were 
developed by using single plant selection method their characteristic and origin are given in Table 1. This method is regarded as the most effective for varietal development in safflower (Singh \& Nimbkar 2007). According to the method, individual plants of each genotype were selected in terms of some morphological and agronomic parameters (flower colour, spines, earliness, plant height, head number per plant and number of seed per head) correlated to seed yield and oil content (Khidir 1974; Patil et al 1994; Malleshappa et al 2003; Alizadeh 2005; Omidi et al 2012; Rudolphi et al 2012). Each year selected single plants replanted next year as a new generation. When the selection reached $\mathrm{S}_{7}$, the plots were harvested in bulk and genotype evaluated screening nursery especially seed yield, oil content and oil yield. Material selection was ended in $\mathrm{S}_{8}$.

Experimental location has typically a steppe climate with temperature differences between day and night and dries in summer, relatively rainy winter. The weather conditions during the experimental period are presented in Table 2. Soil is clayey and neutral in reaction. It is poor in organic matter and reach in available potassium and phosphorus content. Trials were set up in randomized complete block design with three replications; $80 \mathrm{~kg} \mathrm{ha}^{-1}$ nitrogen and $60 \mathrm{~kg} \mathrm{ha}^{-1}$ phosphorus were applied at seeding. Plots were $5 \mathrm{~m}$ long, with $45 \mathrm{~cm}$ between rows and $5-10 \mathrm{~cm}$ between plants within rows after thinning. A length of $45 \mathrm{~cm}$ on both sides of the

Table 1- Some morphological characteristics of the genotypes

\begin{tabular}{llllllll}
\hline Genotype & Line/Variety & Flower colour & Spines & Genotype & Line/Variety & Flower colour & Spines \\
\hline GE-36-2 & Line & Yellow & Spiny & GE-36-25 & Line & Yellow-Orange & Spiny \\
GE-36-3 & Line & Yellow-Orange & Spiny & GE-36-26 & Line & Yellow-Orange Spiny \\
GE-36-4 & Line & Yellow-Orange & Spiny & GE-36-27 & Line & Yellow & Spiny \\
GE-36-6 & Line & Yellow & Spiny & GE-36-28 & Line & Yellow-Orange Spiny \\
GE-36-7 & Line & Yellow & Spiny & GE-36-29 & Line & Yellow & Spiny \\
GE-36-8 & Line & Yellow & Spiny & GE-36-30 & Line & Yellow-Orange Spiny \\
GE-36-9 & Line & Yellow-Orange & Spiny & GE-36-34 & Line & Yellow-Orange Spiny \\
GE-36-10 & Line & Yellow & Spiny & GE-36-36 & Line & Yellow-Orange Spiny \\
GE 36-11 & Line & Yellow-Orange & Spiny & BALCI & Variety & Yellow & Spiny \\
GE-36-12 & Line & Yellow & Spiny & REMZIBEY & Variety & Yellow-Orange Spiny \\
GE-36-13 & Line & Yellow-Orange & Spiny & DINÇER & Variety & Orange-Red & Spineless \\
GE-36-14 & Line & Yellow-Orange & Spiny & YENICE & Variety & Red & Spineless \\
GE-36-17 & Line & Yellow & Spiny & & & & \\
\hline
\end{tabular}

Table 2- Monthly and growing season mean temperature, rainfall and relative humidity in 2010,2011 and 2012

\begin{tabular}{|c|c|c|c|c|c|c|c|c|c|c|c|c|}
\hline \multirow[b]{2}{*}{ Month } & \multicolumn{4}{|c|}{ Mean temperature $\left({ }^{\circ} \mathrm{C}\right)$} & \multicolumn{4}{|c|}{ Rainfall $(\mathrm{mm})$} & \multicolumn{4}{|c|}{ Relative humidity (\%) } \\
\hline & $\begin{array}{c}\text { Long } \\
\text { term }\end{array}$ & 2010 & 2011 & 2012 & $\begin{array}{c}\text { Long } \\
\text { term }\end{array}$ & 2010 & 2011 & 2012 & $\begin{array}{c}\text { Long } \\
\text { term }\end{array}$ & 2010 & 2011 & 2012 \\
\hline March & 4.9 & 5.9 & 3.7 & 1.5 & 33.4 & 32.6 & 20.0 & 56.4 & 82.3 & 85.5 & 88.0 & 87.7 \\
\hline April & 10.4 & 9.2 & 7.2 & 12.0 & 35.2 & 23.9 & 56.9 & 22.1 & 85.6 & 84.3 & 91.0 & 72.6 \\
\hline May & 14.9 & 15.2 & 0.5 & 14.4 & 43.3 & 20.7 & 145.8 & 80.9 & 75.9 & 70.4 & 87.7 & 83.3 \\
\hline June & 18.9 & 18.1 & 16.6 & 20.0 & 28.6 & 79 & 9.4 & 0 & 80.4 & 82.8 & 84.6 & 71.6 \\
\hline July & 21.5 & 22 & 21.6 & 22.8 & 13.5 & 7.4 & 8.5 & 5.5 & 69.2 & 75.4 & 70.8 & 68.1 \\
\hline August & 21.1 & 24.4 & 20.0 & 20.8 & 6.4 & 0.9 & 0 & 3.5 & 71.2 & 66.2 & 73.5 & 65.1 \\
\hline Mean & 15.3 & 15.8 & 11.6 & 15.3 & - & - & - & - & 77.4 & 77.4 & 82.6 & 74.7 \\
\hline Total & - & - & - & - & 160.4 & 164.5 & 240.6 & 168.4 & - & - & - & - \\
\hline
\end{tabular}


rows in each plot was left as border effects. Date of planting is made in the month of March every three years. The trial was performed under natural conditions without irrigation. During growing season weeds were controlled by hand. Plants were harvested in August.

Samples of each plot were obtained to determine seed yield $\left(\mathrm{kg} \mathrm{ha}^{-1}\right)$, number of heads per plant (number), head diameter $(\mathrm{cm}), 1000$ seed weight $(\mathrm{g})$, oil content $(\%)$ and oil yield per hectare $(\mathrm{kg}$ $\mathrm{ha}^{-1}$ ). Oil content of genotype was determined by using Soxhlet apparatus. Oil yield was calculated by multiplying oil content and the seed yield of each plot. Analysis of variance (ANOVA) was performed with the statistical package JMP 5.0.1 (SAS 1989-
2002). Statistically significant differences among the mean values were determined with the least significant difference (LSD) test at the 0.05 level.

\section{Result and Discussion}

In this study, significant differences were determined between the genotypes for all the traits investigated in three years of the study and according to the three-year combined analysis, which implied genetic variation existed for these traits. The analysis demonstrated that significant difference existed between the years expect for head diameter. Year $\mathrm{x}$ genotype interaction was found important for all the traits investigated (Table 3, 4 and 5). It is because that there was some environmental factor in

Table 3- Mean values and statistics group of seed yield and number of head per plant studied some safflower lines and varieties in 2010, 2011, 2012 and 2010-2012

\begin{tabular}{|c|c|c|c|c|c|c|c|c|}
\hline \multirow{2}{*}{ Genotype $e^{\S}$} & \multicolumn{4}{|c|}{ Seed yield $\left(\mathrm{kg} \mathrm{ha}^{-1}\right)$} & \multicolumn{4}{|c|}{ Number of head per plant } \\
\hline & 2010 & 2011 & 2012 & $2010-12$ & 2010 & 2011 & 2012 & $2010-12$ \\
\hline GE-ES-YA-2 & $1390.0 \mathrm{a}-\mathrm{f}$ & $1841.7 \mathrm{c}-\mathrm{g}$ & $1180.5 \mathrm{c}-\mathrm{f}$ & $1470.2 \mathrm{f}-1$ & $11.2 \mathrm{~b}-\mathrm{f}$ & $10.8 \mathrm{f}-\mathrm{j}$ & $9.6 \mathrm{~b}-\mathrm{g}$ & $10.5 \mathrm{~d}-1$ \\
\hline GE-ES-YA-3 & $1330.9 \mathrm{a}-\mathrm{f}$ & $1682.6 \mathrm{~d}-\mathrm{g}$ & $1260.5 \mathrm{~cd}$ & $1420.9 \mathrm{~g}-\mathrm{j}$ & $10.7 \mathrm{c}-\mathrm{f}$ & $11.4 \mathrm{~d}-1$ & $10.6 \mathrm{a}-\mathrm{d}$ & $10.9 \mathrm{c}-\mathrm{g}$ \\
\hline GE-ES-YA-4 & 1490.8 a-c & 1920.8 b-g & $1560.7 \mathrm{ab}$ & $1660.2 \mathrm{~b}-\mathrm{f}$ & $11.0 \mathrm{~b}-\mathrm{f}$ & $12.9 \mathrm{a}-\mathrm{f}$ & $11.2 \mathrm{ab}$ & $11.7 \mathrm{~b}-\mathrm{d}$ \\
\hline GE-ES-YA-6 & $1450.2 \mathrm{a}-\mathrm{d}$ & $1723.1 \mathrm{~d}-\mathrm{g}$ & $1600.0 \mathrm{a}$ & $1590.2 \mathrm{c}-\mathrm{g}$ & $12.0 \mathrm{a}-\mathrm{c}$ & $11.9 \mathrm{~b}-\mathrm{h}$ & $11.7 \mathrm{a}$ & $11.9 \mathrm{a}-\mathrm{c}$ \\
\hline GE-ES-YA-7 & $1530.9 \mathrm{ab}$ & $2327.6 \mathrm{a}-\mathrm{c}$ & $1630.0 \mathrm{a}$ & $1830.2 \mathrm{ab}$ & $11.4 \mathrm{~b}-\mathrm{e}$ & $13.4 \mathrm{a}-\mathrm{f}$ & $10.0 \mathrm{~b}-\mathrm{f}$ & $11.6 \mathrm{~b}-\mathrm{d}$ \\
\hline GE-ES-YA-8 & $1280.1 \mathrm{c}-\mathrm{g}$ & $2117.7 b-d$ & $1130.7 \mathrm{c}-\mathrm{f}$ & $1510.2 \mathrm{~d}-\mathrm{h}$ & $10.5 \mathrm{c}-\mathrm{f}$ & $14.2 \mathrm{a}-\mathrm{c}$ & $9.4 \mathrm{~d}-\mathrm{h}$ & $11.4 \mathrm{~b}-\mathrm{e}$ \\
\hline GE-ES-YA-9 & $1300.2 \mathrm{c}-\mathrm{g}$ & $1728.0 \mathrm{~d}-\mathrm{g}$ & 1020.0 d-g & 1350.0 h-k & $11.0 \mathrm{~b}-\mathrm{f}$ & $11.7 \mathrm{c}-\mathrm{h}$ & $7.9 \mathrm{~h}$ & $10.2 \mathrm{e}-1$ \\
\hline GE-ES-YA-10 & 1410.1 a-e & $1893.1 \mathrm{~b}-\mathrm{g}$ & $1240.3 \mathrm{c}-\mathrm{e}$ & 1510.6 d-h & $12.1 \mathrm{a}-\mathrm{c}$ & $10.8 \mathrm{e}-\mathrm{j}$ & $8.2 \mathrm{~g}-\mathrm{h}$ & $10.4 \mathrm{e}-1$ \\
\hline GE-ES-YA-11 & $1360.0 \mathrm{a}-\mathrm{f}$ & 2042.8 b-e & $1000.0 \mathrm{e}-\mathrm{g}$ & $1460.8 \mathrm{f}-1$ & $10.6 \mathrm{c}-\mathrm{f}$ & $12.8 \mathrm{a}-\mathrm{g}$ & $8.9 \mathrm{e}-\mathrm{h}$ & $10.8 \mathrm{c}-\mathrm{h}$ \\
\hline GE-ES-YA-12 & 1440.0 a-d & $2658.6 \mathrm{a}$ & $1280.0 \mathrm{~cd}$ & $1790.3 \mathrm{a}-\mathrm{c}$ & $12.6 \mathrm{ab}$ & $14.8 \mathrm{a}$ & $9.3 \mathrm{~d}-\mathrm{h}$ & $12.2 \mathrm{ab}$ \\
\hline GE-ES-YA-13 & $1370.3 \mathrm{a}-\mathrm{f}$ & $2403.0 \mathrm{ab}$ & 1020.5 d-g & $1600.1 \mathrm{c}-\mathrm{g}$ & $13.2 \mathrm{a}$ & $14.4 \mathrm{ab}$ & $9.2 \mathrm{~d}-\mathrm{h}$ & $12.3 \mathrm{ab}$ \\
\hline GE-ES-YA-14 & 1400.4 a-e & $2388.3 \mathrm{ab}$ & $1270.3 \mathrm{~cd}$ & $1680.9 \mathrm{~b}-\mathrm{e}$ & $13.2 \mathrm{a}$ & $14.9 \mathrm{a}$ & $11.0 \mathrm{a}-\mathrm{c}$ & $13.0 \mathrm{a}$ \\
\hline GE-ES-YA-17 & $1180.1 \mathrm{fg}$ & 2034.8 b-e & $960.7 \mathrm{fg}$ & $1390.4 \mathrm{~g}-\mathrm{j}$ & $9.7 \mathrm{fg}$ & $12.6 \mathrm{a}-\mathrm{g}$ & $8.5 \mathrm{f}-\mathrm{h}$ & $10.3 \mathrm{e}-1$ \\
\hline GE-ES-YA-25 & $730.2 \mathrm{~h}$ & $2081.5 \mathrm{~b}-\mathrm{d}$ & $850.7 \mathrm{~g}$ & $1220.3 \mathrm{jk}$ & $8.7 \mathrm{~g}$ & $12.6 \mathrm{a}-\mathrm{g}$ & 8.9 e-h & $10.1 \mathrm{f}-1$ \\
\hline GE-ES-YA-26 & $1080.7 \mathrm{~g}$ & $1405.1 \mathrm{~g}$ & $980.0 \mathrm{fg}$ & $1150.8 \mathrm{k}$ & $9.9 \mathrm{e}-\mathrm{g}$ & $10.2 \mathrm{~g}-\mathrm{j}$ & $8.2 \mathrm{~g}-\mathrm{h}$ & 9.41 \\
\hline GE-ES-YA-27 & $1260.1 \mathrm{~d}-\mathrm{g}$ & $1536.8 \mathrm{e}-\mathrm{g}$ & $1070.3 \mathrm{c}-\mathrm{g}$ & $1290.01-\mathrm{k}$ & $10.2 \mathrm{~d}-\mathrm{g}$ & $9.7 \mathrm{~h}-\mathrm{j}$ & $9.6 \mathrm{~b}-\mathrm{g}$ & $9.8 \mathrm{~g}-1$ \\
\hline GE-ES-YA-28 & $1500.0 \mathrm{a}-\mathrm{c}$ & $2333.6 \mathrm{a}-\mathrm{c}$ & $1320.0 \mathrm{bc}$ & $1710.8 \mathrm{~b}-\mathrm{d}$ & $10.9 \mathrm{c}-\mathrm{f}$ & $14.3 \mathrm{a}-\mathrm{c}$ & $9.9 \mathrm{~b}-\mathrm{f}$ & $11.7 \mathrm{~b}-\mathrm{d}$ \\
\hline GE-ES-YA-29 & $1300.3 \mathrm{c}-\mathrm{g}$ & $1817.0 \mathrm{c}-\mathrm{g}$ & $1300.7 \mathrm{bc}$ & $1470.6 \mathrm{f}-1$ & $11.8 \mathrm{a}-\mathrm{d}$ & $13.7 \mathrm{a}-\mathrm{d}$ & $9.4 \mathrm{~d}-\mathrm{h}$ & $11.6 \mathrm{~b}-\mathrm{d}$ \\
\hline GE-ES-YA-30 & $1320.1 \mathrm{~b}-\mathrm{f}$ & $1935.4 \mathrm{~b}-\mathrm{f}$ & $1240.7 \mathrm{c}-\mathrm{e}$ & $1500.1 \mathrm{e}-\mathrm{h}$ & $11.0 \mathrm{~b}-\mathrm{f}$ & $13.5 \mathrm{a}-\mathrm{e}$ & $8.7 \mathrm{e}-\mathrm{h}$ & $11.1 \mathrm{~b}-\mathrm{f}$ \\
\hline GE-ES-YA-34 & $1290.0 \mathrm{c}-\mathrm{g}$ & 2029.6 b-e & $1140.4 \mathrm{c}-\mathrm{f}$ & 1480.8 e-1 & $10.3 \mathrm{~d}-\mathrm{g}$ & $8.3 \mathrm{j}$ & $10.2 \mathrm{a}-\mathrm{e}$ & $9.6 \mathrm{~h}-1$ \\
\hline GE-ES-YA-36 & $1560.0 \mathrm{a}$ & $2683.9 \mathrm{a}$ & $1740.0 \mathrm{a}$ & $1990.5 \mathrm{a}$ & $12.0 \mathrm{a}-\mathrm{c}$ & $13.1 \mathrm{a}-\mathrm{f}$ & $10.3 \mathrm{a}-\mathrm{e}$ & $11.8 \mathrm{bc}$ \\
\hline YENİCE & $1210.7 \mathrm{e}-\mathrm{g}$ & $1446.7 \mathrm{f}-\mathrm{g}$ & $1110.7 \mathrm{c}-\mathrm{g}$ & $1260.0 \mathrm{jk}$ & $10.9 \mathrm{~b}-\mathrm{f}$ & $8.9 \mathrm{ij}$ & 9.9 b-f & $9.9 \mathrm{f}-1$ \\
\hline DİNÇER & $1400.4 \mathrm{a}-\mathrm{f}$ & 2031.9 b-e & $1090.7 \mathrm{c}-\mathrm{g}$ & $1510.1 \mathrm{e}-\mathrm{h}$ & $11.9 \mathrm{a}-\mathrm{d}$ & $13.8 \mathrm{a}-\mathrm{d}$ & 10.3 a-e & $12.0 \mathrm{a}-\mathrm{c}$ \\
\hline REMZİBEY & $1340.2 \mathrm{a}-\mathrm{f}$ & $1793.3 \mathrm{~d}-\mathrm{g}$ & 1000.0 e-g & $1370.8 \mathrm{~h}-\mathrm{j}$ & $11.6 \mathrm{a}-\mathrm{d}$ & $11.8 \mathrm{~b}-\mathrm{h}$ & $9.4 \mathrm{c}-\mathrm{h}$ & $11.0 \mathrm{c}-\mathrm{g}$ \\
\hline BALCI & $1340.0 \mathrm{a}-\mathrm{f}$ & $2113.3 \mathrm{~b}-\mathrm{d}$ & $1200.7 \mathrm{c}-\mathrm{f}$ & $1550.3 \mathrm{~d}-\mathrm{h}$ & $11.1 \mathrm{~b}-\mathrm{f}$ & $11.7 \mathrm{c}-\mathrm{h}$ & $9.8 \mathrm{~b}-\mathrm{g}$ & $10.9 \mathrm{cg}$ \\
\hline Mean & 1330.3 & 1990.9 & 1210.1 & 1510.4 & 11.2 & 12.3 & 9.6 & 11.0 \\
\hline Genotype & $* *$ & $* *$ & $* *$ & ** & * & $* *$ & * & $* *$ \\
\hline Year & & & & $* *$ & & & & $*$ \\
\hline Genotype x Year & & & & ** & & & & $* *$ \\
\hline
\end{tabular}


Table 4- Mean values and statistics group of head diameter and 1000 seed weight studied some safflower lines and varieties in 2010, 2011, 2012 and 2010-2012

\begin{tabular}{|c|c|c|c|c|c|c|c|c|}
\hline \multirow{2}{*}{ Genotype $^{\S}$} & \multicolumn{4}{|c|}{ Head diameter $(\mathrm{cm})$} & \multicolumn{4}{|c|}{1000 seed weight $(g)$} \\
\hline & 2010 & 2011 & 2012 & $2010-12$ & 2010 & 2011 & 2012 & $2010-12$ \\
\hline GE-ES-YA-2 & $2.33 \mathrm{~b}-\mathrm{g}$ & $2.17 \mathrm{gh}$ & $2.63 \mathrm{a}-\mathrm{d}$ & $2.38 \mathrm{c}-\mathrm{g}$ & $42.8 \mathrm{~d}-1$ & $47.5 \mathrm{a}-\mathrm{d}$ & $44.5 \mathrm{c}-\mathrm{f}$ & $45.0 \mathrm{c}-1$ \\
\hline GE-ES-YA-3 & $2.60 \mathrm{ab}$ & $2.17 \mathrm{gh}$ & $2.27 \mathrm{e}-\mathrm{g}$ & $2.34 \mathrm{e}-\mathrm{g}$ & $41.7 \mathrm{f}-\mathrm{j}$ & $45.0 \mathrm{~b}-\mathrm{f}$ & $43.4 \mathrm{~d}-\mathrm{f}$ & $43.4 \mathrm{~g}-\mathrm{j}$ \\
\hline GE-ES-YA-4 & $2.33 \mathrm{~b}-\mathrm{g}$ & $2.45 b-f$ & $2.87 \mathrm{ab}$ & $2.55 \mathrm{bc}$ & $39.8 \mathrm{~h}-\mathrm{k}$ & $43.8 \mathrm{e}-\mathrm{h}$ & $39.5 \mathrm{ij}$ & $41.0 \mathrm{kl}$ \\
\hline GE-ES-YA-6 & $2.13 \mathrm{f}-1$ & $2.38 \mathrm{~d}-\mathrm{g}$ & $2.20 \mathrm{~g}$ & $2.24 \mathrm{~g}$ & $43.2 \mathrm{c}-\mathrm{h}$ & 46.5 a-e & $47.9 \mathrm{ab}$ & $45.9 \mathrm{c}-\mathrm{e}$ \\
\hline GE-ES-YA-7 & $2.10 \mathrm{~g}-1$ & $2.40 \mathrm{c}-\mathrm{g}$ & $2.23 \mathrm{fg}$ & $2.24 \mathrm{~g}$ & $47.8 \mathrm{ab}$ & $47.6 \mathrm{a}-\mathrm{d}$ & $48.4 \mathrm{a}$ & $47.9 \mathrm{ab}$ \\
\hline GE-ES-YA-8 & $2.30 \mathrm{c}-\mathrm{h}$ & $2.40 \mathrm{c}-\mathrm{g}$ & $2.63 \mathrm{a}-\mathrm{d}$ & $2.44 \mathrm{c}-\mathrm{f}$ & $42.2 \mathrm{e}-\mathrm{j}$ & $45.7 \mathrm{a}-\mathrm{f}$ & $44.5 \mathrm{c}-\mathrm{f}$ & $44.1 \mathrm{e}-\mathrm{j}$ \\
\hline GE-ES-YA-9 & $2.20 \mathrm{e}-1$ & $2.40 \mathrm{c}-\mathrm{g}$ & $2.70 \mathrm{a}-\mathrm{c}$ & $2.43 \mathrm{c}-\mathrm{f}$ & $39.31-\mathrm{k}$ & $44.6 \mathrm{c}-\mathrm{f}$ & $44.2 \mathrm{~d}-\mathrm{f}$ & $42.7 \mathrm{jk}$ \\
\hline GE-ES-YA-10 & $2.62 \mathrm{a}$ & $2.80 \mathrm{a}$ & $2.90 \mathrm{a}$ & $2.77 \mathrm{a}$ & $39.0 \mathrm{jk}$ & $48.6 \mathrm{ab}$ & $47.3 \mathrm{a}-\mathrm{c}$ & $45.0 \mathrm{c}-\mathrm{h}$ \\
\hline GE-ES-YA-11 & $2.10 \mathrm{~g}-1$ & $2.57 \mathrm{a}-\mathrm{d}$ & $2.33 \mathrm{~d}-\mathrm{g}$ & $2.33 \mathrm{e}-\mathrm{g}$ & $45.3 \mathrm{a}-\mathrm{e}$ & $49.1 \mathrm{a}$ & 45.8 a-e & $46.7 \mathrm{a}-\mathrm{c}$ \\
\hline GE-ES-YA-12 & $2.40 \mathrm{a}-\mathrm{f}$ & $2.27 \mathrm{e}-\mathrm{h}$ & $2.70 \mathrm{a}-\mathrm{c}$ & $2.46 \mathrm{c}-\mathrm{e}$ & $43.5 \mathrm{c}-\mathrm{g}$ & 47.6 a-d & $47.4 \mathrm{a}-\mathrm{c}$ & $46.2 \mathrm{~b}-\mathrm{d}$ \\
\hline GE-ES-YA-13 & $2.03 \mathrm{~h} 1$ & $2.40 \mathrm{c}-\mathrm{g}$ & $2.27 \mathrm{e}-\mathrm{g}$ & $2.23 \mathrm{~g}$ & $46.5 \mathrm{a}-\mathrm{c}$ & $45.2 b-f$ & 45.6 a-e & $45.8 \mathrm{c}-\mathrm{f}$ \\
\hline GE-ES-YA-14 & $2.13 \mathrm{f}-1$ & $2.10 \mathrm{~h}$ & $2.47 \mathrm{c}-\mathrm{g}$ & $2.23 \mathrm{~g}$ & $46.4 \mathrm{a}-\mathrm{d}$ & $47.9 \mathrm{a}-\mathrm{c}$ & 45.9 a-d & $46.7 \mathrm{a}-\mathrm{c}$ \\
\hline GE-ES-YA-17 & $2.17 \mathrm{e}-1$ & $2.50 \mathrm{~b}-\mathrm{e}$ & $2.57 \mathrm{~b}-\mathrm{e}$ & $2.41 \mathrm{c}-\mathrm{g}$ & $41.1 \mathrm{f}-\mathrm{k}$ & 45.9 a-e & $44.6 \mathrm{c}-\mathrm{f}$ & $43.9 \mathrm{f}-\mathrm{j}$ \\
\hline GE-ES-YA-25 & $2.10 \mathrm{~g}-1$ & $2.20 \mathrm{f}-\mathrm{h}$ & $2.53 \mathrm{c}-\mathrm{f}$ & $2.27 \mathrm{fg}$ & $34.4 \mathrm{~lm}$ & $44.1 \mathrm{~d}-\mathrm{g}$ & $42.7 \mathrm{e}-\mathrm{h}$ & 40.41 \\
\hline GE-ES-YA-26 & $2.20 \mathrm{e}-1$ & $2.53 \mathrm{~b}-\mathrm{d}$ & $2.49 \mathrm{c}-\mathrm{g}$ & $2.41 \mathrm{c}-\mathrm{g}$ & $41.3 \mathrm{f}-\mathrm{k}$ & $42.2 \mathrm{f}-\mathrm{h}$ & 39.7 h-j & $41.1 \mathrm{kl}$ \\
\hline GE-ES-YA-27 & $2.19 \mathrm{e}-1$ & $2.27 \mathrm{e}-\mathrm{h}$ & $2.53 \mathrm{c}-\mathrm{g}$ & $2.33 \mathrm{e}-\mathrm{g}$ & $42.3 \mathrm{e}-\mathrm{j}$ & $46.2 \mathrm{a}-\mathrm{e}$ & $45.3 \mathrm{~b}-\mathrm{f}$ & $44.6 \mathrm{~d}-\mathrm{j}$ \\
\hline GE-ES-YA-28 & $2.23 \mathrm{~d}-1$ & $2.50 \mathrm{~b}-\mathrm{e}$ & $2.43 \mathrm{c}-\mathrm{g}$ & $2.39 \mathrm{c}-\mathrm{g}$ & 40.0 g-k & $45.8 \mathrm{a}-\mathrm{f}$ & 45.9 a-d & 43.9 e-j \\
\hline GE-ES-YA-29 & $2.21 \mathrm{e}-1$ & $2.27 \mathrm{e}-\mathrm{h}$ & $2.60 \mathrm{a}-\mathrm{d}$ & $2.36 \mathrm{~d}-\mathrm{g}$ & $39.31-\mathrm{k}$ & $40.8 \mathrm{gh}$ & $40.2 \mathrm{~g}-\mathrm{j}$ & 40.11 \\
\hline GE-ES-YA-30 & $2.40 \mathrm{a}-\mathrm{f}$ & $2.40 \mathrm{c}-\mathrm{g}$ & $2.50 \mathrm{c}-\mathrm{g}$ & $2.43 \mathrm{c}-\mathrm{f}$ & $32.5 \mathrm{~m}$ & $40.2 \mathrm{~h}$ & $35.7 \mathrm{k}$ & $36.2 \mathrm{~m}$ \\
\hline GE-ES-YA-34 & $2.53 \mathrm{a}-\mathrm{c}$ & $2.53 \mathrm{~b}-\mathrm{d}$ & $2.90 \mathrm{a}$ & $2.66 \mathrm{ab}$ & $39.1 \mathrm{jk}$ & $43.6 \mathrm{e}-\mathrm{h}$ & $38.8 \mathrm{jk}$ & 40.51 \\
\hline GE-ES-YA-36 & 2.001 & $2.70 \mathrm{ab}$ & $2.33 \mathrm{~d}-\mathrm{g}$ & $2.34 \mathrm{e}-\mathrm{g}$ & $48.0 \mathrm{a}$ & $48.9 \mathrm{a}$ & $48.3 \mathrm{ab}$ & $48.4 \mathrm{a}$ \\
\hline YENİCE & $2.30 \mathrm{c}-\mathrm{h}$ & $2.63 \mathrm{a}-\mathrm{c}$ & $2.67 \mathrm{a}-\mathrm{c}$ & $2.53 \mathrm{~b}-\mathrm{d}$ & $38.0 \mathrm{kl}$ & $48.2 \mathrm{a}-\mathrm{c}$ & $42.9 \mathrm{~d}-\mathrm{g}$ & $43.01-\mathrm{k}$ \\
\hline DİNÇER & $2.42 \mathrm{a}-\mathrm{e}$ & $2.50 \mathrm{~b}-\mathrm{e}$ & $2.50 \mathrm{c}-\mathrm{g}$ & $2.47 \mathrm{~b}-\mathrm{e}$ & $44.2 b-f$ & 45.90 a-e & $45.3 \mathrm{a}-\mathrm{f}$ & $45.1 \mathrm{c}-\mathrm{g}$ \\
\hline REMZİBEY & $2.50 \mathrm{a}-\mathrm{d}$ & $2.43 \mathrm{c}-\mathrm{f}$ & $2.50 \mathrm{c}-\mathrm{g}$ & $2.48 \mathrm{~b}-\mathrm{e}$ & $41.2 \mathrm{f}-\mathrm{k}$ & $45.67 \mathrm{a}-\mathrm{f}$ & $42.3 \mathrm{f}-1$ & $43.1 \mathrm{~h}-\mathrm{j}$ \\
\hline BALCI & $2.40 \mathrm{a}-\mathrm{f}$ & $2.53 \mathrm{~b}-\mathrm{d}$ & $2.70 \mathrm{a}-\mathrm{c}$ & $2.54 \mathrm{bc}$ & $40.6 \mathrm{~g}-\mathrm{k}$ & $44.80 \mathrm{c}-\mathrm{f}$ & 45.7 a-e & $43.7 \mathrm{~g}-\mathrm{j}$ \\
\hline Mean & 2.28 & 2.42 & 2.54 & 2.41 & 41.6 & 45.7 & 44.1 & 43.8 \\
\hline Genotype & $* *$ & $* *$ & $* *$ & $* *$ & $* *$ & $* *$ & $* *$ & $* *$ \\
\hline Year & & & & ns & & & & $*$ \\
\hline Genotype x Year & & & & $*$ & & & & $*$ \\
\hline
\end{tabular}

$\S$, means in the same column followed by the same letters were not significantly different at 0.05 level using LSD test; * and **, significant at the 5 and $1 \%$ level, respectively; ns, not significant

year effect which was responsible for differences in cultivar responses to this trait. Means of seed yield, number of head per plant, head diameter, 1000 seed weight, oil content, oil yield were found 1330.31990.9 and $1210.1 \mathrm{~kg} \mathrm{ha}^{-1}, 11.2-12.3$ and 9.6 number plant $^{-1}, 2.28-2.42$ and $2.54 \mathrm{~cm}, 41.6-45.7$ and 44.1 g, 36.1-36.6 and $35.6 \%, 470.9-730.0$ and $430.0 \mathrm{~kg}$ ha $^{-1}$ in 2010, 2011 and 2012, respectively (Table 3, 4 and 5).

\subsection{Seed yield}

To increase seed yield is one of the main aim in breeding research of field crops and it is also important to determine high yielding genotype in safflower. Environmental condition is as important as genotype on grain yield, therefore breeders want to tested performance lines and varieties developed in different years and location. 
When the results examined, mean of seed yield were found $1330.3 \mathrm{~kg} \mathrm{ha}^{-1}$ in $2010,1990.9 \mathrm{~kg} \mathrm{ha}^{-1}$ in 2011, $1210.1 \mathrm{~kg} \mathrm{ha}^{-1}$ in 2012 (Table 3). It was determined that the second year had higher seed yield than the others. The reason is that experimental location took high rainfall, especially, during the early stages of development in 2011 (Table 2). It was emphasized that seed yield was significantly affected water supply and rain, particularly during early stage of safflower (Agasimani et al 1997; Uslu et al 2002).

Considering grain yield mean values, the highest seed yield was obtained from line GE-36-36 all years of the study and three-year averages and this line gave $1560.0 \mathrm{~kg} \mathrm{ha}^{-1}, 2683.9 \mathrm{~kg} \mathrm{ha}^{-1}$ and $1740.0 \mathrm{~kg}$ $\mathrm{ha}^{-1}$ seed yield in 2010, 2011 and 2012, respectively. Based on the average of the combined values of three years, GE-ES-YA-36-36 (1990.5 kg ha-1), GEES-YA-36-7 (1830.2 $\left.\mathrm{kg} \mathrm{ha}^{-1}\right)$, GE-ES-YA-36-12 $\left(1790.3 \mathrm{~kg} \mathrm{ha}^{-1}\right)$ belonged to the same group, with respect to seed yield (Table 3). To consider of this trial value of the varieties; Balci reached $1550.3 \mathrm{~kg}$ $\mathrm{ha}^{-1}$ and Dincer (1510.1 $\left.\mathrm{kg} \mathrm{ha}^{-1}\right)$, Remzibey (1370.8 $\mathrm{kg} \mathrm{ha}^{-1}$ ) and Yenice (126.0 $\left.\mathrm{kg} \mathrm{ha}^{-1}\right)$ followed. When the result examined, large number of lines passed the varieties in terms of seed yield. Genotype and ecological factors are two major factors, which are influential on seed yield. Beside of this seed yield was the most affected trait from the environment (Camas \& Esendal 2006). The evaluation of seed yield values demonstrated that variations existed between the lines and varieties. These variations were considered may have been from the reactions of the different cultivars and lines to different ecological conditions. It was reported that, seed yield varied 1164.0-2810.0 kg ha' $\mathrm{kg}^{-1}$ Bergman et al (1989); 920.0-1050.0 kg ha-1 Muralidharudu \& Nagaraj (1990); 1030.0-1250.0 kg ha-1 Bayraktar (1995); 1300.0-2700.0 kg ha-1 Reinbrecht et al (2005); 1330.0-2394.0 kg ha-1 Koutroubas \& Papadoska (2005); 1107.5-1823.8 $\mathrm{kg} \mathrm{ha}^{-1}$ Camas et al (2007); 1706.0-3111.0 kg ha ${ }^{-1}$ Kizil et al (2008); 971.0$1585.0 \mathrm{~kg} \mathrm{ha}^{-1}$ Beyyavas et al (2011) and 1602.0$2167.0 \mathrm{~kg} \mathrm{ha}^{-1}$ Zarei et al (2011). In this research differences and similarities between the results of the previous researches referred above and also in this trial might be concerned with genetic diversity of the genotypes, different ecological condition and agronomic application.

\subsection{Yield components}

In breeding programs, new varieties developed are selected by using various yield components are used to determine seed yield (Omidi et al 2012). Although the number of head per plant affected by environmental conditions, it is important trait influence the yield (Consentino et al 1997; Omidi Tabrizi 2000; Beyyavas et al 2011). Weiss (2000) is reported to obtain high seed yield, well-developed 12 to 14 heads per plant are sufficient. In this research, number of heads per plant ranged between 8.7 and 13.2 in first year, 8.3 and 14.9 in second year, 7.9 and 11.7 in third year of the study (Table 3 ). The three-year averages, number of heads per plant ranged between 9.4 number plant ${ }^{-1}$ (GEES-YA-36-26) and 13.0 number plant ${ }^{-1}$ (GE-ESYA-36-14). Dajue \& Mündel (1996) emphasize that number of heads per plant is very strongly linked to yield in safflower. Chaudhary (1990) showed that number of heads per plant could be used determined to high seed yielding varieties with 50 safflower lines. The highest number of heads per plant had been obtained by the researchers were Cazzato et al (2001), 30.0 number plant $^{-1}$; Beyyavas et al (2011), 19.5 number plant $^{-1}$ and Ada (2013) 23.7 number plant $^{-1}$ in their research. The investigated result in this trial showed differences between previous data. This is the result of different environmental condition and agronomic application. Zarei et al (2011) reported that number of head per plant greatly affected from environmental conditions particularly plant density.

The result of variances analysis examined there is no significant difference existed between the years for head diameter. These result showed that head diameter is not effect different year condition whereas significantly varied with genotypes. According to the Table 4 average of the combined values of three years, head diameter of the lines and the varieties ranged between $2.23 \mathrm{~cm}$ (GE-ES- 
YA-36-13) and $2.77 \mathrm{~cm}$ (GE-ES-YA-36-10). Ashri et al (1976) were reported that head diameter can vary considerably without affecting seed yield; studied on safflower germplasm collection consisted of 900 lines.

When the results examined, 1000 seed weight of genotype ranged between $32.5-48.0 \mathrm{~g}$ in 2010, 40.2 and $49.1 \mathrm{~g}$ in 2011, 35.7 and $48.4 \mathrm{~g}$ in 2012 (Table 4). The genotypes investigated in this study, displayed significant variation. This diversity was considered to reaction of genotypes to different ecological conditions in this study. Beyyavas et al (2011) reported that genetic structure and ecological factors are two major factors, which are influential on 1000 seed weight. Acharya et al (1994) reported that 1000 -seed weight had positively effect on seed yield and also indicated the influence of this character was greater than the other characters in

Table 5- Mean values and statistics group of oil content and oil yield studied some safflower lines and varieties in 2010, 2011, 2012 and 2010-2012

\begin{tabular}{|c|c|c|c|c|c|c|c|c|}
\hline \multirow[b]{2}{*}{ Genotype $^{\S}$} & \multicolumn{4}{|c|}{ Oil content (\%) } & \multicolumn{4}{|c|}{ Oil yield $\left(\mathrm{kg} \mathrm{ha}^{-1}\right)$} \\
\hline & 2010 & 2011 & 2012 & $2010-12$ & 2010 & 2011 & 2012 & $2010-12$ \\
\hline GE-ES-YA-2 & $37.0 \mathrm{ef}$ & $36.6 \mathrm{~d}-\mathrm{h}$ & $37.3 \mathrm{~d}-\mathrm{f}$ & $37.0 \mathrm{~cd}$ & 510.4 a-e & $670.4 \mathrm{~b}-\mathrm{h}$ & $440.4 \mathrm{c}-\mathrm{g}$ & $540.4 \mathrm{c}-1$ \\
\hline GE-ES-YA-3 & 36.4 e-h & $37.3 \mathrm{~b}-\mathrm{g}$ & $35.4 \mathrm{~h} 1$ & $36.4 \mathrm{~d}-\mathrm{f}$ & $480.6 \mathrm{~b}-\mathrm{f}$ & $620.6 \mathrm{e}-1$ & $440.8 \mathrm{~b}-\mathrm{g}$ & $520.0 \mathrm{e}-\mathrm{j}$ \\
\hline GE-ES-YA-4 & $38.6 \mathrm{a}-\mathrm{d}$ & $39.9 a-b$ & $37.3 \mathrm{~d}-\mathrm{f}$ & $38.6 \mathrm{a}$ & $570.8 \mathrm{a}$ & $760.7 \mathrm{a}-\mathrm{g}$ & $580.4 \mathrm{a}$ & $640.3 \mathrm{ab}$ \\
\hline GE-ES-YA-6 & $35.1 \mathrm{~h} 1$ & $36.4 \mathrm{~d}-1$ & $33.8 \mathrm{k}-\mathrm{m}$ & $35.1 \mathrm{gh}$ & 500.9 a-e & $620.6 \mathrm{e}-1$ & $540.0 \mathrm{ab}$ & $550.9 \mathrm{c}-\mathrm{g}$ \\
\hline GE-ES-YA-7 & $35.9 \mathrm{f}-\mathrm{h}$ & $36.5 \mathrm{~d}-\mathrm{h}$ & 35.21 & $35.9 \mathrm{e}-\mathrm{g}$ & $550.2 \mathrm{ab}$ & $840.8 \mathrm{a}-\mathrm{c}$ & $570.4 \mathrm{a}$ & $650.8 \mathrm{a}$ \\
\hline GE-ES-YA-8 & $37.4 \mathrm{~b}-\mathrm{e}$ & $38.3 \mathrm{a}-\mathrm{d}$ & $36.6 \mathrm{fg}$ & $37.4 \mathrm{~b}-\mathrm{c}$ & $470.9 \mathrm{~b}-\mathrm{f}$ & 810.2 a-e & $410.6 \mathrm{c}-\mathrm{k}$ & $560.9 \mathrm{~b}-\mathrm{f}$ \\
\hline GE-ES-YA-9 & $35.4 \mathrm{~g}-1$ & $36.1 \mathrm{~d}-\mathrm{j}$ & $34.8 \mathrm{ij}$ & $35.4 \mathrm{fg}$ & $460.1 \mathrm{c}-\mathrm{g}$ & 620.4 e-1 & $350.5 \mathrm{~g}-\mathrm{k}$ & $480.0 \mathrm{~h}-\mathrm{j}$ \\
\hline GE-ES-YA-10 & $35.8 \mathrm{f}-\mathrm{h}$ & $35.0 \mathrm{f}-\mathrm{j}$ & $36.6 \mathrm{fg}$ & $35.8 \mathrm{e}-\mathrm{g}$ & 500.6 a-e & $660.3 \mathrm{c}-\mathrm{h}$ & $450.6 \mathrm{~b}-\mathrm{e}$ & $540.2 \mathrm{c}-1$ \\
\hline GE-ES-YA-11 & $37.4 \mathrm{c}-\mathrm{e}$ & $38.6 \mathrm{a}-\mathrm{d}$ & $36.1 \mathrm{gh}$ & $37.4 \mathrm{~b}-\mathrm{d}$ & 500.8 a-e & 790.0 a-f & $360.1 \mathrm{f}-\mathrm{k}$ & $550.3 \mathrm{c}-\mathrm{h}$ \\
\hline GE-ES-YA-12 & $34.11-\mathrm{k}$ & $34.0 \mathrm{~h}-\mathrm{k}$ & $34.2 \mathrm{jk}$ & $34.1 \mathrm{~h} 1$ & 490.2 b-f & $900.5 \mathrm{a}$ & $430.8 \mathrm{c}-\mathrm{h}$ & $610.1 \mathrm{a}-\mathrm{d}$ \\
\hline GE-ES-YA-13 & $34.2 \mathrm{ij}$ & $34.7 \mathrm{~g}-\mathrm{j}$ & $33.7 \mathrm{k}-\mathrm{m}$ & $34.2 \mathrm{~h} 1$ & $470.0 \mathrm{c}-\mathrm{f}$ & $830.4 \mathrm{a}-\mathrm{d}$ & 340.6 h-k & $550.0 \mathrm{c}-1$ \\
\hline GE-ES-YA-14 & $32.8 \mathrm{kl}$ & 34.2 h-k & $31.4 \mathrm{o}$ & $32.8 \mathrm{j}$ & $460.0 \mathrm{c}-\mathrm{g}$ & 810.9 a-e & $400.0 \mathrm{c}-\mathrm{k}$ & $560.0 \mathrm{c}-\mathrm{g}$ \\
\hline GE-ES-YA-17 & $38.8 \mathrm{ab}$ & $40.3 \mathrm{a}$ & $37.3 \mathrm{~d}-\mathrm{f}$ & $38.8 \mathrm{a}$ & $450.8 \mathrm{c}-\mathrm{g}$ & 820.2 a-e & $360.0 \mathrm{f}-\mathrm{k}$ & $540.7 \mathrm{c}-1$ \\
\hline GE-ES-YA-25 & $39.2 \mathrm{a}$ & $40.4 \mathrm{a}$ & $38.0 \mathrm{a}-\mathrm{c}$ & $39.2 \mathrm{a}$ & $280.8 \mathrm{~h}$ & 830.9 a-d & $320.5 \mathrm{jk}$ & $480.4 \mathrm{~g}-\mathrm{j}$ \\
\hline GE-ES-YA-26 & $39.2 \mathrm{a}$ & 39.9 a-c & $38.5 \mathrm{a}$ & $39.2 \mathrm{a}$ & $420.5 \mathrm{fg}$ & 550.9 h1 & $370.7 \mathrm{~d}-\mathrm{k}$ & $450.4 \mathrm{jk}$ \\
\hline GE-ES-YA-27 & $38.4 \mathrm{a}-\mathrm{d}$ & 37.8 a-e & $39.0 \mathrm{a}$ & $38.4 \mathrm{ab}$ & 480.4 b-f & $580.0 \mathrm{~g}-1$ & $410.8 c-j$ & $490.4 \mathrm{f}-\mathrm{j}$ \\
\hline GE-ES-YA-28 & $35.7 \mathrm{f}-\mathrm{h}$ & $37.3 \mathrm{c}-\mathrm{g}$ & $34.1 \mathrm{j}-1$ & $35.7 \mathrm{e}-\mathrm{g}$ & $530.5 \mathrm{a}-\mathrm{c}$ & $870.2 \mathrm{ab}$ & $450.1 \mathrm{~b}-\mathrm{f}$ & $610.9 \mathrm{a}-\mathrm{c}$ \\
\hline GE-ES-YA-29 & $36.5 \mathrm{e}-\mathrm{g}$ & $35.4 \mathrm{e}-\mathrm{j}$ & $37.6 \mathrm{c}-\mathrm{e}$ & $36.5 \mathrm{c}-\mathrm{e}$ & $470.4 \mathrm{~b}-\mathrm{f}$ & $640.0 \mathrm{~d}-\mathrm{h}$ & $490.1 \mathrm{a}-\mathrm{c}$ & $530.5 \mathrm{c}-1$ \\
\hline GE-ES-YA-30 & $39.3 \mathrm{a}$ & $40.3 \mathrm{a}$ & $38.3 \mathrm{ab}$ & $39.3 \mathrm{a}$ & $520.0 \mathrm{a}-\mathrm{d}$ & $770.9 \mathrm{a}-\mathrm{g}$ & $470.8 \mathrm{bc}$ & 590.2 a-e \\
\hline GE-ES-YA-34 & $37.3 \mathrm{de}$ & $37.6 b-f$ & 37.0 ef & $37.3 \mathrm{~cd}$ & $480.2 \mathrm{~b}-\mathrm{f}$ & $760.3 \mathrm{a}-\mathrm{g}$ & $420.4 \mathrm{c}-1$ & $550.6 \mathrm{c}-\mathrm{h}$ \\
\hline GE-ES-YA-36 & $33.6 \mathrm{jk}$ & $33.81-\mathrm{k}$ & $33.31-\mathrm{m}$ & $33.6 \mathrm{ij}$ & $520.4 \mathrm{a}-\mathrm{c}$ & $900.3 \mathrm{a}$ & $570.9 \mathrm{a}$ & $660.9 \mathrm{a}$ \\
\hline YENİCE & $31.5 \mathrm{~lm}$ & 30.01 & $33.0 \mathrm{mn}$ & $31.5 \mathrm{k}$ & $380.3 \mathrm{~g}$ & 430.41 & 360.8 e-k & $390.5 \mathrm{k}$ \\
\hline DİNÇER & $31.2 \mathrm{~m}$ & $32.1 \mathrm{kl}$ & $30.3 \mathrm{p}$ & $31.2 \mathrm{k}$ & $430.8 \mathrm{e}-\mathrm{g}$ & $650.2 \mathrm{c}-\mathrm{h}$ & $330.21-\mathrm{k}$ & $470.4 \mathrm{ij}$ \\
\hline REMZİBEY & $33.0 \mathrm{jk}$ & $33.7 \mathrm{jk}$ & 32.2 no & $33.0 \mathrm{j}$ & $440.2 \mathrm{~d}-\mathrm{g}$ & $600.6 \mathrm{f}-1$ & $320.2 \mathrm{k}$ & $450.7 \mathrm{jk}$ \\
\hline BALCI & $38.7 \mathrm{a}-\mathrm{c}$ & $38.3 \mathrm{a}-\mathrm{d}$ & $39.0 \mathrm{a}$ & $38.7 \mathrm{a}$ & $510.6 \mathrm{a}-\mathrm{e}$ & 810.1 a-e & $470.1 \mathrm{~b}-\mathrm{d}$ & $590.9 \mathrm{a}-\mathrm{d}$ \\
\hline Mean & 36.1 & 36.6 & 35.6 & 36.1 & 470.9 & 730.0 & 430.0 & 540.7 \\
\hline Genotype & $* *$ & $* *$ & $* *$ & $* *$ & $* *$ & $* *$ & $* *$ & $* *$ \\
\hline Year & & & & $* *$ & & & & $* *$ \\
\hline Genotype x Year & & & & $*$ & & & & $* *$ \\
\hline
\end{tabular}

$\S$, means in the same column followed by the same letters were not significantly different at 0.05 level using LSD test; * and **, significant at the 5 and $1 \%$ level, respectively 
relation to seed yield. According to average of three years, GE-ES-YA-36-36 (48.4 g), GE-ES-YA-36-7 (47.9 g), GE-ES-YA-36-14 and GE-ES-YA-36-11 $(46.7 \mathrm{~g})$ belonged to the same statistic group in terms of 1000 seed weight. The first two lines were also included in the first group with the highest seed yield. These results showed that 1000 weight seed was reliable components influencing seed yield.

\subsection{Oil content}

The value of safflower oil is dramatically increased nowadays, economically, oil content of seed is important for safflower production and considered as the major factors, affecting the success of safflower introduction in new production areas (Vorpsi et al 2010). Therefore, improving seed yield as well as the oil content of safflower has become importance in breeding program. According to this research, oil content of genotype ranged between $31.2 \%$ and $39.3 \%$ in the first year, $30.0 \%$ and $40.4 \%$ in the second year, $30.3 \%$ and $39.0 \%$ in the third year of the study (Table 5). Compared the lines and the varieties, it was observed that oil content of lines increased and most of them exceed the varieties. This situation is the result of selection made in terms of oil content along breeding program and the difference between the lines and varieties is strongly due to the genotype, beside of environmental condition. Consentino et al (1997), Johnson et al (1999), Uysal et al (2006), Zhang \& Chen (2005), Koutroubas \& Papadoska (2005), Gawand et al (2005), Arslan \& Kucuk (2005) and Kose (2013) were reported that oil content varied between 33.4$43.4 \%$; $13-46 \% ; 23.7-26.9 \% ; 23.8-40.3 \%$; 26.7$35.7 \% ; 26.3-28.5 \% ; 31.3-36.3 \%$ and $30.6-38.7 \%$, respectively. These results showed some similarity and differences in our results depend on genotype, trial and environmental condition. Hang \& Evans (1985) reported that oil content mostly depends on the genotype, beside of this climatic factor and agronomic practices also affected it (Esendal \& Tosun 1972; Pascal Villalobos \& Alburquerque 1996; Rahamatalla et al 2001). When the combined values examined GE-ES-YA-36-30 (39.3\%), GEES-YA-36-25 and GE-ES-YA-36-26 (39.2\%), GE-
ES-YA-36-17 (38.8\%), GE-ES-YA-36-4 (38.6\%), GE-ES-YA-36-27 (38.4\%) had the first statistic group and remarkable in terms of oil content.

\subsection{Oil yield}

The oil yield calculated on the seed yield and oil content of genotypes, affected by these two factors. When the examined the result, oil yield of genotype ranged between 280.8 and $570.8 \mathrm{~kg} \mathrm{ha}^{-1}$ in 2010 , 430.4 and $900.5 \mathrm{~kg} \mathrm{ha}^{-1}$ in 2011, 320.2 and 580.4 $\mathrm{kg} \mathrm{ha}^{-1}$ in 2012 (Table 5). It is clear that highest oil yield obtained in the second year, similar in seed yield. This result revealed that the increase of oil yield was primarily associated with the increase of seed yield. According to average of three years, lines GE-ES-YA-36-36 with $660.9 \mathrm{~kg} \mathrm{ha}^{-1}$, GE-ESYA-36-7 (650.8 kg ha $\left.{ }^{-1}\right)$, GE-ES-YA-36-4 (640.3 $\left.\mathrm{kg} \mathrm{ha}^{-1}\right)$, GE-ES-YA-36-28 (610.9 kg ha $\left.{ }^{-1}\right)$, GE-ESYA-36-12 (610.1 kg ha-1) and variety Balci (590.9 $\mathrm{kg} \mathrm{ha}^{-1}$ ) were in the same statistic group, with respect to higher oil yield per hectare. The result from the present study indicated that oil yield of safflower has been affected of genotype oil content, seed yield and ecologic conditions under which the experiments were carried out. For oil yield, the previous studies were recorded such data as 390.6-514.4 $\mathrm{kg} \mathrm{ha}^{-1}$, Rajput et al (2007); 322 to 460 $\mathrm{kg} \mathrm{ha}^{-1}$ Gawand et al (2005) and 416-701 kg ha-1 Koutroubas \& Papadoska (2005). The finding of the present study is in parallel with the results of other researchers.

\section{Conclusions}

This study indicated that large number of line had higher performance than varieties in terms of yield, yield components, oil content and oil yield. In this case, the result of selection of lines showed superior performance compared to the varieties since $\mathrm{S}_{5}$ generation.

Based on all three years and combined analysis results of this study, lines GE-ES-YA-36-36, GEES-YA-36-7 in terms of seed yield, lines GE-ESYA-36-30, GE-ES-YA-36-25, GE-ES-YA-36-26, GE-ES-YA-36-27 in terms of oil content and 
lines GE-ES-YA-36-36, GE-ES-YA-36-7, GEES-YA-36-4 in terms of oil yield were listed at the highest statistical group. Consequently, it was decided that these lines could be candidate varieties with regard to these different characteristics.

\section{References}

Acharya S, Dhaduk L K \& Maliwal G L (1994). Path analysis in safflower (Carthamus tinctorius L.) under conserved moisture conditions. Gujarat Agricultural University Research Journal 20: 154-157

Ada R (2013). Cluster analysis and adaptation study for safflower genotypes. Bulgarian Journal of Agricultural Science 19: 103-109

Agasimani C A, Patil R \& Hand Radder G E (1997). Recent advances in agronomy of safflower (C. tinctorius L.) in India. In: Proceedings of $I V^{\text {th }}$ International Safflower Conference, 2-7 June, Bari, Italy, pp. 77-82

Alizadeh K (2005). Evaluation of safflower germplasm by some agronomic characteristics and their relationships on grain yield production in the cold dry land of Iran. International Journal of Agriculture Biology 3: 389391

Arslan B \& Kucuk M (2005). Oil content and fatty acid composition of some safflower cultivars in Van, Turkey. In: Proceedings of VI $I^{\text {th }}$ International Safflower Conference, 6-10 June, Istanbul, Turkey, pp. 167-175

Ashri A, Urie A L \& Zimmer D E (1976). Evaluation of the world collection of safflower, Carthamus tinctorius $L$. variability of capitulum width and outerin volucral bract dimension. Euphytica 25: 225-229

Bayraktar N (1995). The seed yield and yield component in the third progeny of safflower hybrids. Journal of Field Crops Central Research Institute 1: 23-29

Bergman J W \& Charles R F (2008). Evaluation of safflower and other oil seed crops grown in the United States Northern Plains region for biofuels/biobased products. In: Proceedings of VII ${ }^{\text {th }}$ International Safflower Conference, 3-6 November, Wagga Wagga, Australia

Bergman J, Carlson G, Kushnak G, Riveland N R, Stallknecht G, Welty L E \& Wichman D (1989). Registration of "Finch" safflower. Crop Science 29: 829

Beyyavas V, Haliloglu H, Copur O \& Yilmaz A (2011). Determination of seed yield and yield components of some safflower (Carthamus tinctorius L.) cultivars, lines and populations under the semi-arid conditions. African Journal of Biotechnology 10: 527-534

Camas N \& Esendal E (2006). Estimates of broad sense heritability for seed and yield components of safflower. Hereditas 143: 55-57

Camas N, Cirak C \& Esendal E (2007). Seed yield, oil content and fatty acids composition of safflower (Carthamus tinctorius L.) grown in Northern Turkey Conditions. Journal of Faculty of Agriculture 22(1): 98-104

Cazzato E, Borazio L \& Corleto A (2001). Grain yield, oil content and earliness of flowering of hybrids and openpollinated safflower in southern Italy. In: Proceedings of $V^{\text {th }}$ International Safflower Conference, 23-27 July, Wiliston, U.S.A, pp. 185-189

Chaudhary S K (1990). Path analysis for seed yield in safflower (Carthamus tinctorius L.) in acid soil under mid altitude conditions. International Journal Tropical Agriculture 8: 129-132

Consentino S L, Copani V, Cammarat M \& Riggi E (1997). Relations between metereological parameters, yield and seed oil content in safflower (Carthamus tinctorius L.) in Mediterranean environment. In: Proceedings of $I V^{\text {th }}$ International Safflower Conference, 2-7 June, Bari, Italy, pp. 149-152

Dajue L \& Mündel H (1996). Safflower (Carthamus tinctorius L.) promoting the conservation and use of underutilized and neglected crops. International Plant Genetic Resources Institute, Rome, Italy, pp. 1-83

El-Lattief E A (2012). Evaluation of 25 safflower genotypes for seed and oil yields under arid environment in upper Egypt. Asian Journal of Crop Science 4: 72-79

Esendal E \& Tosun F (1972). Research on the yield, phonological, morphological and seed characteristics of some native and foreign varieties of safflower (Carthamus tinctorius L.) under Erzurum Ecological Conditions. Atatürk University Journal of Faculty of Agriculture 3: 93-115

Falconer D S \& Mackay T F C (1981). Introduction to Quantitative Genetics, Longman. Harlow, UK

FAO (2014). The Food and Agriculture Organization of the United Nations. http://faostat.fao.org/site/567/ default.aspx\#ancor (Access date: 05.05.2015)

Fernandez Martinez J, Dominguez Gimenez J, Jimenez A \& Hernandez L (1986). Use of the single seed descent method in breeding safflower (Carthamus tinctorius L.). Plant Breeding 97: 289-378 
Gawand P B, Reddyand B N \& Tambe S I (2005). Evaluation of productivity of safflower cultivars under moisture and nutrient management in rainfeed vertisols. In: Proceedings of $V I^{\text {th }}$ International Safflower Conference, 6-10 June, Istanbul, Turkey, pp. 205-209

Hang A N \& Evans D W (1985). Deficit sprinkler irrigation of sunflower and safflower. Agronomy Journal 77: 588-592

Johnson R C, Bergman J W \& Flynn C R (1999). Oil and meal characteristics of core and non-core safflower accessions from the USDA collection. Genetic Resources and Crop Evolution 46: 611-618

Khidir M O (1974). Genetic variability and interrelationship of some quantitative characters in safflower. The Journal of Agricultural Science 83: 177-202

Kizil S, Cakmak O, Kirici S \& Inan M (2008). A comprehensive study on safflower (Carthamus tinctorius L.) in semi-arid conditions. Biotechnology \& Biotechnological Equipment 22(4): 947-953

Koutroubas S D \& Papadoska D K (2005). Adaptation, grain yield and oil content of safflower in Greece. In: Proceedings of $V I^{\text {th }}$ International Safflower Conference, 6-10 June, Istanbul, Turkey, pp. 161-167

KoseA(2013). Research on yield and quality characteristics of some safflower (Carthamus tinctorius L.) lines and varieties under dry and irrigated conditions in Turkey. Journal of Food Agriculture \& Environment 11(1): 553-556

Kose A, Hatipoglu H, Kose T F, Arslan H, Kosar F \& Karakus M (2011). Determining the performances in Eskisehir and Sanliurfa conditions. In: GAP $V I^{\text {th }}$ Agriculture Congress, 9-12 May, Sanliurfa, Turkey, pp. 286-291

Malleshappa S M, Hiremath I \& Ravikumar R I (2003). Negative associations between important quantitative traits in safflower (Carthamus tinctorius L.). Sesame and Safflower Newsletter 18: 80-84

Muralidharudu Y \& Nagaraj H (1990). Effect of location and genotype on safflower oil and its quality. Field Crops Abstracts 43(5): 3516

Mündel H H (2008). Major achievements in safflower breeding and future challenges. In: Proceedings of Products, VII In International Safflower Conference, 3-6 November, Wagga Wagga, Australia

Omidi A H, Hassan A, Khazaei H, Monneveux P \& Stoddard F (2012). Effect of cultivar and water regime on yield and yield components in safflower (Carthamus tinctorius L.). Turkish Journal of Field Crops 17: 10-15

Omidi Tabrizi A H (2000). Correlation between characters and path analysis for grain and oil yield in spring safflower. Sesame and Safflower Newsletter 15: 78-80

Pascal Villalobos M J \& Alburquerque N (1996). Genetic variation of a safflower germplasm collection grown as a winter crop in Southern Spain. Euphytica 3: 327332

Patil V D, Reddy M V S \& Nerkar Y S (1994). Efficiency of early generation selections for yield and related characters in safflower (Carthamus tinctorius L.). Theorotical and Applied Genetics 89: 293-296

Poehlman, M J (1978). Breeding Field Crops. The Avi Publishing Company, U.S.A

Rahamatalla A B, Babiker E E, Krishna A G \& El-Tinay A $H$ (2001). Changes in fatty acids composition during seed growth and physicochemical characteristics of oil extracted from four safflower cultivars. Plant Foods Human Nutrient 56: 385-395

Rajput G R, Khawale V S, Mankar P S, Sharma S K \& Kumbhare M D (2007). Effect of sowing dates and nutrient management in safflower. Journal of Soils and Crops 17(1): 110-113

Reinbrecht C, Barth S, VonWithzke-Ehbrecht S, Frick C, Elfadl E, Kahnt G, Becker H C \& Claupein W (2005). Screening of a worldwide safflower collection adaptation to humid temperate climates and cultivation in organic farming. In: Proceedings of VIth International Safflower Conference, 6-10 June, Istanbul, Turkey, pp. 236-242

Rudolphi S, Becker H C, Schierholt A \& Witzke-Ehbrecht $S$ (2012). Improved estimation of oil, linoleic and oleic acid and seed hull fractions in safflower by NIRS. Journal of American Oil Chemists Society 89: 363-369

Shinwari Z K, Rehman H \& Rabbani M A (2014). Morphological traits based genetic diversity in safflower. Pakistan Journal of Botany 46(4): 13891395

Singh V \& Nimbkar N (2007). Safflower (Carthamus tinctorius L.). In: R J Singh (Ed), Genetic Recources Chomosome Engineering and Crop Improvement, CRC Press, Baco Raton, pp. 167-194

Sujatha M (2008). Biotechnological interventions for genetic improvement of safflower. In: Proceedings 
of VII International Safflower Conference, 3-6 November, Wagga Wagga, Australia

TSI (2014). Turkish Statistical Institute. http://www.tuik. gov.tr/bitkiselap/bitkisel.zul. (Access date: 05.05.2015)

Uher J (2008). Safflower in European Floriculture. In: Proceeding of VII ${ }^{\text {th }}$ International Safflower Conference, 3-6 November, Wagga Wagga, Australia

Uslu N, Tutluer I, Taner Y, Kunter B, Sagel Z \& Peskircioglu H (2002). Effects of temperature and moisture stress during elongation and branching on development and yield of safflower. Sesame and Safflower Newsletter 17: 117-122

Uysal N, Baydar H \& Erbas S (2006). Determination of agricultural and technological properties of safflower (Carthamus tinctorus L.) lines developed from Isparta population. Suleyman Demirel University Journal of Faculty of Agriculture 1(1): 52-63

Vorpsi V, Harizaj F, Bardhi N, Vladi V \& Dodona E (2010). Carthamus tinctorius L, the quality of safflower seeds cultivated in Albania. Research Journal of Agricultural Science 42(1): 326-331

Zarei G, Shamsi H \& Fazeli F (2011). Effect of planting density on yield and yield components of safflower cultivars in spring. International Scholarly and Scientific Research \& Innovation 5(12): 118-120

Zhang Z \& Chen Y (2005). Studies on adaptability of safflower germplasms in Xinjiang China. In: Proceedings of $I V^{\text {th }}$ International Safflower Conference, 6-10 June, Istanbul, Turkey, pp. 132-139

Weiss E A (2000). Safflower. In: Oilseed Crops, Blackwell Science, Victoria, Australia, pp. 93-129 